\title{
Erratum to: An Analysis of Gravitational Redshift from Rotating Body
}

\section{Anuj Kumar Dubey • A. K. Sen}

Published online: 19 February 2015

(C) Springer Science+Business Media New York 2015

\section{Erratum to: Int J Theor Phys \\ DOI 10.1007/s10773-014-2464-3}

The above authors wish to correct a typographical error found in the original article. Equation (62) has writing mistake. The correct equation is found below:

$$
\omega^{\prime}=-\frac{\partial \Psi}{\partial x^{0}} \frac{\partial x^{0}}{\partial \tau}-\frac{\partial \Psi}{\partial x^{\phi}} \frac{\partial x^{\phi}}{\partial \tau}=\omega \frac{\partial x^{0}}{c \partial \tau}-k_{\phi} u^{\phi}=\omega u^{0}-k_{\phi} u^{\phi}
$$

instead of

$$
\omega^{\prime}=-\frac{\partial \Psi}{\partial x^{0}} \frac{\partial x^{0}}{\partial \tau}=\omega \frac{\partial x^{0}}{c \partial \tau}=\omega u^{0}
$$

The online version of the original article can be found at http://dx.doi.org/10.1007/s10773-014-2464-3.

A. K. Dubey $(\bowtie) \cdot$ A. K. Sen

Department of Physics, Assam University, Silchar, 788011, Assam, India e-mail: danuj67@gmail.com

\section{A. K. Sen}

e-mail: asokesen@yahoo.com 